\title{
FATORES DE RISCO E EMOCIONAIS NA VOZ DE PROFESSORES COM E SEM QUEIXAS VOCAIS
}

\section{The risk factors and emotional on the voice of teachers with and without vocal complaints}

\author{
Denise Batista da Costa ${ }^{(1)}$, Leonardo Wanderley Lopes(2), Eveline Gonçalves Silva ${ }^{(3)}$, \\ Germana Maria Soares da Cunha ${ }^{(4)}$, Larissa Nadjara Alves Almeida( ${ }^{(5)}$, Anna Alice Figueirêdo de Almeida( ${ }^{(6)}$
}

\begin{abstract}
RESUMO
Objetivo: analisar a interferência dos fatores de riscos e emocionais na voz de professores com e sem queixa. Método: a amostra foi composta de 44 professores. Utilizou-se uma ficha com dados pessoais e profissionais, três questionários referentes aos aspectos vocais e dois questionários para dados relacionados à emoção, sobretudo ansiedade, além da coleta de voz para análise perceptivo-auditiva. Os grupos foram divididos a partir do número de sintomas relatados, estabelecendo um número de 22 voluntários no grupo de Professores Sem Queixa (PSQ) e 22 no grupo Professores Com Queixas (PCQ), sendo deste grupo, os indivíduos que relataram mais de três sintomas vocais. Os dados foram analisados estatisticamente. Resultados: a média do número de sintomas descritos no QSSV foi de 5,7 $( \pm 2,8)$ para os $P C Q$ e de $0,8( \pm 0,9)$ para os $P S Q$. Os $P S Q$ referiram uma melhor autoavaliação vocal $(p=0,01)$ e os $P C Q$ afirmaram possuir um maior comprometimento em sua voz $(p=0,001)$. O grupo PSQ obteve os maiores valores nos escores do QVV-Físico $(p=0,0007)$ e QVV-Total $(p=0,0006)$. Os PCQ obtiveram maiores valores no IDV-Total $(p=0,0003)$ e IDV-Orgânico $(p=0,0006)$, e um maior comprometimento emocional, com SRQ de 5,7 $( \pm 3,9)$ e IDATE $42,5( \pm 12,7)$. A partir da avaliação perceptivo-auditiva, os PCQ apresentaram um desvio vocal moderado com presença de rugosidade, soprosidade e tensão na voz, enquanto os PSQ evidenciam um desvio leve em todos os parâmetros. Conclusão: os professores com queixas vocais estão expostos a mais fatores de riscos, além de relatarem mais sintomas e comprometimento vocais, bem como emocionais.
\end{abstract}

DESCRITORES: Docentes; Voz; Ansiedade; Emoções; Comunicação; Fonoaudiologia

(1) Acadêmica do Curso de Graduação de Fonoaudiologia pela Universidade Federal da Paraíba - UFPB; Bolsista de Iniciação científica do CNPq.

(2) Fonoaudiólogo; Docente do Departamento de Fonoaudiologia da Universidade Federal da Paraíba - UFPB; Doutor em Linguística pela Universidade Federal da Paraíba - UFPB.

(3) Acadêmica do Curso de Graduação de Fonoaudiologia pela Universidade Federal da Paraíba - UFPB; Bolsista de Iniciação científica do CNPq.

(4) Acadêmica do Curso de Graduação de Fonoaudiologia pela Universidade Federal da Paraíba - UFPB; Voluntária do Programa de Iniciação Científica da UFPB.

(5) Acadêmica do Curso de Graduação de Fonoaudiologia pela Universidade Federal da Paraíba - UFPB; Voluntária do Programa de Iniciação Científica da UFPB.

(6) Fonoaudióloga; Docente do Departamento de Fonoaudiologia da Universidade Federal da Paraíba - UFPB; Doutora em Ciências pela Universidade Federal de São Paulo - UNIFESP.

Auxílio Financeiro: $\mathrm{CNPq}$

Conflito de interesses: inexistente

\section{INTRODUÇÃO}

A Fonoaudiologia vem se dedicando ao estudo da disfonia ocupacional vinculada a professores, muito em razão do crescente número de pedidos de afastamento do trabalho relacionados a problemas envolvendo a voz. Os professores compõem uma das classes mais afetadas por problemas vocais e várias são as consequências provocadas, como a dificuldade de desenvolver a profissão até problemas relacionados à comunicação, vida social e emocional ${ }^{1}$.

Sabe-se que a voz é o instrumento primordial para a atuação dos docentes em sala de aula para que se concretize o processo de ensino-aprendizagem. Qualquer desvio que ocorra nesse instrumento pode afetar a atuação profissional dos 
professores e comprometer a aprendizagem dos alunos. Entretanto, muitos desses profissionais não valorizam os sintomas relacionados à disfonia, agravando, assim, seu problema. Esse fato torna mais difícil sua recuperação, o que pode acarretar no afastamento do seu exercício profissional².

A disfonia caracteriza-se pela presença de sintomas vocais e pode ser de origem orgânica e/ ou funcional, sendo os sintomas principais: cansaço ao falar, pigarro, rouquidão, ardência na garganta, falhas na voz, falta de ar para falar, dentre outros ${ }^{3}$.

Em pesquisa recente ${ }^{4}$, verificou-se que a maioria dos professores apresentou qualidade vocal alterada, sendo o grau moderado o mais frequente. Queixas específicas pareceram se distribuir igualmente entre professoras com e sem alteração de voz. Por fim, os pesquisadores apontaram a necessidade de projetos de saúde vocal para esta população.

Diversos fatores interferem para que se desenvolva uma desordem vocal em professores. Esses estão relacionados ao ambiente de trabalho, excesso de atividades e a pressão imposta pelas instâncias superiores, o que pode gerar estresse e ansiedade, componentes que também se associam aos problemas na voz. Assim, vê-se que problemas emocionais podem estar envolvidos na causa ou consequência do problema vocal ${ }^{1}$.

Alguns estudiosos da área apontam a interferência das emoções na produção da voz. Afirmam que o indivíduo expressa em sua voz traços da personalidade, sentimentos, estado de saúde, humor, depressão, frustrações, dentre outros. A ansiedade e o estresse causam algumas alterações fisiológicas no corpo, assim, as estruturas que compõem o trato vocal também são afetadas, modificando a emissão da voz ${ }^{5}$.

Deve-se ressaltar que o inverso também ocorre. Os distúrbios vocais podem causar estresse psicoemocional, depressão e frustração, que afetam negativamente o funcionamento social, além de causar um impacto significante na qualidade de vida e na eficiência do trabalho do indivíduo ${ }^{6,7}$.

Em pesquisa recente ${ }^{8}$, observou-se que quanto maior o grau de ansiedade traço, maior número de sinais e sintomas vocais, além de haver maior comprometimento da comunicação e da qualidade de vida em diversos aspectos relacionados ao uso da voz.

Diante das questões apresentadas, viu-se a necessidade de conhecer qual a relação entre aspectos emocionais, sobretudo ansiedade, e problemas vocais em professores. Além disso, existindo essa relação, precisa-se compreender se ela é de casualidade ou de efeito, de manutenção ou de gênese da disfonia, em pessoas que apresentam comprometimento emocional.

Assim, o objetivo deste artigo é analisar a interferência dos fatores de riscos e emocionais na voz de professores com e sem queixa vocal.

\section{MÉTODO}

Esta é uma pesquisa quantitativa, de campo, do tipo observacional transversal. A mesma foi avaliada e aprovada pelo Comitê de Ética em Pesquisa do Hospital Universitário Lauro Wanderley da Universidade Federal da Paraíba, sob protocolo CEP/HULW no 278/09.

Participaram da pesquisa 44 professores, de ambos os sexos, que possuíam entre 18 a 50 anos de idade. Estes pertencentes a cinco escolas do Estado da Paraíba, entre pública e privada.

Este estudo selecionou os voluntários de acordo com os critérios de inclusão de (1) terem a idade entre 18 e 50 anos, pois se entende que o limite inferior garante o processo final de muda vocal e o superior o início de uma série de alterações estruturais na laringe decorrente da senescência, com um maior ou menor impacto vocal ${ }^{9}$; (2) serem Professores com e sem queixas vocais e (3) terem assinado o Termo de Consentimento Livre e Esclarecido (TCLE).

Este estudo teve como critérios de inclusão professores com idade entre 18 e 50 anos, com e sem queixas de voz e que assinaram o Termo de Consentimento Livre e Esclarecido. O limite de 50 anos deu-se considerando-se o risco de alterações da voz em função da idade.

Em relação aos critérios de exclusão, não poderiam participar da pesquisa os indivíduos com doenças neurológicas, além de acometimento das vias aéreas superiores no momento da pesquisa.

Inicialmente, fez-se contato com as escolas, a fim de selecionar locais silentes para a coleta dos dados, bem como a autorização da instituição de ensino para a realização da pesquisa. $\mathrm{Na}$ sequência, os professores foram contatados e sanaram suas dúvidas ao se deparar com o Termo de Consentimento Livre e Esclarecido (TCLE) para que iniciasse a pesquisa.

Em seguida, os professores responderam os cinco questionários relacionados à voz e à emoção. Por fim, coletou-se a amostra de fala em um local silente previamente selecionado.

Os questionários utilizados foram uma ficha com dados pessoais e profissionais (tempo de profissão, carga horária semanal, o tipo de escola, disciplinas lecionadas, existência de ruído no local de trabalho, autopercepção sobre problemas vocais, tratamentos vocais anteriores e uso de medicamentos), 
Questionário de Sinais e Sintomas Vocais (QSSV), Questionário de Qualidade de Vida em Voz (QVV), Índice de Desvantagem Vocal (IDV) para os dados referentes aos aspectos vocais. Para os dados relacionados à emoção, sobretudo ansiedade, utilizou-se o Inventário de Ansiedade TraçoEstado (IDATE) e o Self-Report Questionnaire (SRQ-20). Abaixo, explanou-se brevemente todos os questionários.

\section{Aspectos vocais:}

- Questionário de Sinais e Sintomas Vocais (QSSV)

Contém uma lista com sintomas vocais que indica o aparecimento de um sintoma no passado, no presente, a frequência que ocorre e se relaciona ao trabalho. Tal questionário foi desenvolvido em inglês ${ }^{10}$ e teve uma versão traduzida e adaptada para o português ${ }^{11}$.

- Questionário de Qualidade de Vida em Voz (QVV)

O QVV é um questionário de autoavaliação para mensurar a relação da voz e a qualidade de vida em aspectos relacionados à comunicação, possui 10 itens e 2 domínios: socioemocional, mensurado por meio dos itens 4, 5, 8 e 10; e de funcionamento físico, avaliado por meio das demais questões. O QVV foi traduzido e validado para o português ${ }^{12}$.

- Índice de Desvantagem Vocal (IDV)

É um questionário de autoavaliação para mensurar como um problema vocal interfere nas situações de comunicação diárias. Possui trinta itens distribuídos em três domínios: o funcional, emocional e orgânico. Utilizou-se a versão validada para o português ${ }^{13}$.

\section{Aspectos Emocionais:}

- Inventário de Ansiedade Traço-Estado (IDATE)

Esse instrumento contém duas subescalas com vinte itens e quatro graus de intensidade cada. $\mathrm{O}$ IDATE tem a finalidade de medir o estado subjetivo de ansiedade. Aborda a autopercepção do indivíduo em relação à ansiedade traço, característica de personalidade propensa à ansiedade, e ansiedade estado, condição emocional transitória no momento da aplicação. Utilizou-se sua versão validada para o português ${ }^{14}$.

\section{- Self-Report Questionnaire (SRQ-20)}

Avalia, por meio de 23 questões, distúrbios psiquiátricos, transtornos mentais comuns, principalmente relacionados à ansiedade, depressão e estresse. São considerados emocionalmente comprometidos os homens que assinalarem seis afirmativas e as mulheres, sete. Usou-se a versão validada no Brasil ${ }^{15}$.

Além dos questionários, coletou-se uma amostra vocal, com a finalidade de realizar avaliação perceptivo-auditiva da voz. Para tal, solicitou-se que cada professor emitisse em frequência e intensidade habituais autorreferidas, a vogal sustentada $\mid \varepsilon /$ e uma contagem de 1 a 20. A amostra vocal foi coletada utilizando um microfone headset, da marca Logitech, acoplado a um computador portátil. A amostra vocal foi gravada no software gratuito PRAAT, utilizando-se taxa de amostragem de $44100 \mathrm{~Hz}$.

Para a avaliação perceptivo-auditiva, utilizou-se uma escala analógico-visual (EAV) de 100mm, avaliando os parâmetros grau geral (GG), rugosidade $(R)$, soprosidade $(S)$, tensão $(T)$ e instabilidade $(I)$. A marcação mais próxima do 0 representa menor alteração e quanto mais próxima do 100 , maiores são as alterações. Assim, possui uma possibilidade de gradação de 0 a 100mm a intensidade do desvio vocal.

É importante mencionar que, para a análise dos dados, os professores participantes foram definidos com queixas vocais quando relataram três ou mais sintomas no Questionário de Sinais e Sintomas Vocais (QSSV), sendo alocados no grupo professores com queixas (PCQ), e os que apresentaram um número de sintomas inferior a três foram alocados no grupo professores sem queixas (PSQ).

Os dados coletados foram tabulados em uma planilha eletrônica e, posteriormente, analisados pelo programa estatístico STATISTICA, versão 6.1 , sendo o nível de significância considerado de $\mathrm{p} \leq 0,05$.

Utilizou-se a análise estatística descritiva para descrever as variáveis por meio de média, desvio padrão, frequência e porcentagem, além da estatística inferencial, a partir do teste de Mann Whitney, para comparar o grupo PCQ e PSQ.

\section{RESULTADOS}

Abaixo seguem as tabelas com os resultados da pesquisa e a respectiva discussão desses.

$\mathrm{Na}$ Tabela 1 apresentou-se a frequência da ocorrência de variáveis relacionadas ao trabalho.

Os professores com queixas (PCQ) ministravam disciplinas, predominantemente, da área de Humanas, como História, Geografia e Línguas, já o grupo de professores sem queixas (PSQ) eram responsáveis por outras disciplinas, como informática e artes, seguido da área de Exatas. Alguns professores lecionavam em mais de uma disciplina de diferentes áreas. 
Tabela 1 - Frequência absoluta e percentual de variáveis relacionadas ao trabalho

\begin{tabular}{|c|c|c|c|c|c|}
\hline \multirow{2}{*}{ Variáveis } & \multicolumn{2}{|c|}{$P C Q$} & \multicolumn{2}{|c|}{ PSQ } & \multirow{2}{*}{ Significância } \\
\hline & $\mathbf{N}$ & $\%$ & $\mathbf{N}$ & $\%$ & \\
\hline Disciplinas & & & & & 0,03 \\
\hline Biológicas & 2 & 9,1 & 0 & 0 & \\
\hline Humanas & 8 & 36,4 & 1 & 4,5 & \\
\hline Exatas & 2 & 9,1 & 4 & 18,2 & \\
\hline Línguas & 3 & 13,6 & 4 & 18,2 & \\
\hline Ed. Física & 0 & 0 & 1 & 4,5 & \\
\hline Outras & 4 & 18,2 & 9 & 49,9 & \\
\hline Não Respondeu & 0 & 0 & 1 & 4,5 & \\
\hline Mais de uma área & 3 & 13,6 & 2 & 9,1 & \\
\hline Tipo de Escola & & & & & 0,004 \\
\hline Pública & 8 & 36,4 & 18 & 81,8 & \\
\hline Privada & 6 & 27,2 & 1 & 4,5 & \\
\hline Ambas & 8 & 36,4 & 3 & 13,6 & \\
\hline Relato de ruído no trabalho & & & & & 0,03 \\
\hline Presença & 20 & 90,9 & 14 & 63,6 & \\
\hline Ausência & 2 & 9,01 & 8 & 36,4 & \\
\hline
\end{tabular}

Legenda: PCQ- Professores com queixas; PSQ- Professores sem queixas. Teste Estatístico de Mann Whitney

Ambos os grupos apresentavam predominância no ensino de escolas da rede pública, sendo que a maior parcela se encontrava no grupo PSQ, havendo professores que trabalhavam nos dois tipos de escolas, privada e pública.

O grupo PCQ referia haver mais ruído no ambiente de trabalho, do que o demonstrado pelo $P S Q$, mas ambos os grupos apontavam a presença de ruído nas escolas.

Na Tabela 2, encontram-se as médias e desvios padrão da autoavaliação vocal e emocional dos professores com e sem queixas.

Tabela 2 - Médias e desvios padrão da autoavaliação vocal e emocional dos professores com e sem queixas

\begin{tabular}{lccccc}
\hline \multirow{2}{*}{ Variáveis } & \multicolumn{2}{c}{ PCQ } & \multicolumn{2}{c}{ PSQ } & Significância \\
\cline { 2 - 5 } & Média & $\begin{array}{c}\text { Desvio } \\
\text { Padrão }\end{array}$ & Média & $\begin{array}{c}\text { Desvio } \\
\text { Padrão }\end{array}$ & \\
\hline Números de sintomas & 6,6 & $\pm 2,03$ & 0,8 & $\pm 0,9$ & $<0,0001$ \\
Autoavaliação QSSV & 5,3 & $\pm 1,6$ & 8,8 & $\pm 0,7$ & 0,009 \\
Problema na Voz & 1,4 & $\pm 0,5$ & 1,8 & $\pm 0,4$ & 0,006 \\
Autoavaliação QVV & 3,5 & $\pm 0,8$ & 2,8 & $\pm 0,7$ & 0,006 \\
QVV Físico & 72,9 & $\pm 17,9$ & 91,2 & \pm 10 & 0,0003 \\
QVV Total & 79,5 & $\pm 16,6$ & 94,09 & $\pm 6,7$ & 0,0003 \\
SRQ Total & 5,7 & $\pm 3,9$ & 3,4 & \pm 3 & 0,02 \\
IDATE Estado & 42,5 & $\pm 12,7$ & 37 & $\pm 11,7$ & 0,05 \\
IDV Total & 27,1 & $\pm 22,5$ & 8,9 & $\pm 8,9$ & 0,0003 \\
IDV Orgânico & 16,5 & $\pm 20,4$ & 2,9 & $\pm 3,7$ & $<0,0001$ \\
\hline
\end{tabular}

Legenda: QSSV- Questionário de Sinais e Sintomas Vocais; QVV- Questionário de Qualidade de Vida em Voz; IDATE- Inventário de Ansiedade Traço-Estado; SRQ- Self-Report Questionnaire; IDV- Índice de Desvantagem Vocal. Teste Estatístico de Mann Whitney 
Os $P C Q$ referiram sentir mais sintomas vocais e emocionais e possuíam uma pior autoavaliação da voz comparados com os PSQ, mesmo assim não referiram uma má autopercepção do problema de voz, relatando, menos que o grupo PSQ, a existência do problema.

Para o QVV, os PCQ obtiveram menores escores que os $P S Q$, o que significa que a possível alteração vocal interfere na qualidade de vida dos indivíduos. O domínio físico apresentou-se como o mais comprometido para os $\mathrm{PCQ}$.
Os sintomas emocionais foram mais frequentemente referidos pelos $P C Q$, nos dois questionários para esta avaliação, o SRQ e o IDATE. Apenas o IDATE Estado foi estatisticamente significante, o que expõe um quadro de ansiedade no momento da aplicação da pesquisa.

Os PCQ apresentaram um índice de desvantagem vocal mais elevado que os $\mathrm{PSQ}$, principalmente no domínio orgânico.

Tabela 3 - Ocorrência de sinais e sintomas vocais e emocionais em professores com e sem queixas

\begin{tabular}{lccccc}
\hline \multirow{2}{*}{ Sintomas } & \multicolumn{2}{c}{ PCQ } & \multicolumn{2}{c}{ PSQ } & \multirow{2}{*}{ Significância } \\
\cline { 2 - 5 } & $\mathbf{N}$ & $\%$ & $\mathbf{N}$ & $\%$ & \\
Vocais & 17 & 77,3 & 4 & 18,2 & 0,0002 \\
Problema para cantar agudo & 17 & 77,3 & 4 & 18,2 & 0,0002 \\
Garganta seca & 12 & 54,5 & 3 & 13,6 & 0,01 \\
Gosto ácido na boca & 11 & 50 & 1 & 4,5 & 0,001 \\
Rouquidão & 11 & 50 & 0 & 0 & 0,0003 \\
Voz Cansada & 11 & 50 & 0 & 0 & 0,0003 \\
Problema para falar baixo & 11 & 50 & 1 & 4,5 & 0,0005 \\
Problema para projetar a voz & 11 & 50 & 1 & 4,5 & 0,001 \\
Pigarro & 11 & 50 & 0 & 0 & 0,0002 \\
Desconforto ao falar & 9 & 40,9 & 2 & 9,1 & 0,02 \\
Esforço para falar & 8 & 36,4 & 1 & 4,5 & 0,009 \\
Voz monótona & 6 & 27,3 & 0 & 0 & 0,01 \\
Instabilidade na voz & & & & & 0,002 \\
Emocionais & 14 & 63,6 & 4 & 18,2 & 0,0008 \\
Dor de cabeça & 14 & 63,4 & 3 & 13,6 & 0,03 \\
Má digestão & 11 & 50 & 4 & 18,2 & 0,02 \\
Sensações desagradáveis no estômago & 7 & 31,8 & 1 & 4,5 & \\
Falta de apetite & & & &
\end{tabular}

Teste Estatístico de Mann Whitney

A Tabela 3 expõe a ocorrência de sinais e sintomas vocais e emocionais nos grupos estudados.

Os sintomas vocais e emocionais foram prevalentes no grupo PCQ. Problema para cantar agudo, garganta seca e gosto ácido na boca foram os mais frequentes citados, entre os vocais, e dor de cabeça, má digestão e sensações desagradáveis no estômago, entre os emocionais.
Alguns sintomas abordados nos questionários com o foco na emoção puderam se relacionar com os sintomas dos protocolos destinados à voz, é o caso de má digestão com gosto ácido na boca, sugerindo hábitos alimentares inadequados e/ou alterações no estômago. Assim, certos sintomas emocionais podem ter interferência direta na produção vocal. 
Tabela 4 - Média e desvio padrão da avaliação perceptivo-auditiva da voz de professores com e sem queixas

\begin{tabular}{lccccc}
\hline \multirow{2}{*}{ Variáveis } & \multicolumn{2}{c}{ PCQ } & \multicolumn{2}{c}{ PSQ } & Significância \\
\cline { 2 - 5 } & Média & $\begin{array}{c}\text { Desvio } \\
\text { padrão }\end{array}$ & Média & $\begin{array}{l}\text { Desvio } \\
\text { padrão }\end{array}$ & \\
\hline GG- EAV/É/ & 51 & $\pm 4,1$ & 41,4 & $\pm 2,4$ & $<0,0001$ \\
R-EAV /É/ & 50,5 & \pm 8 & 39,2 & $\pm 6,7$ & $<0,00005$ \\
S-EAV /É/ & 42,5 & \pm 11 & 35,1 & $\pm 12,4$ & $<0,05$ \\
T-EAV /É/ & 49,2 & $\pm 9,5$ & 34 & $\pm 13,5$ & 0,0001 \\
I-EAV /É/ & 51,2 & $\pm 9,8$ & 37,5 & $\pm 12,2$ & 0,0007 \\
GG- EAV Fala & 42,6 & $\pm 10,4$ & 37 & \pm 5 & 0,01 \\
R- EAV Fala & 39,2 & $\pm 16,4$ & 31 & $\pm 14,8$ & 0,05 \\
I-EAV Fala & 34,8 & \pm 20 & 24 & $\pm 14,7$ & 0,04 \\
\hline
\end{tabular}

Legenda: GG- Grau Geral; R- Rugosidade; S- Soprosidade; T- Tensão; I- Instabilidade. Teste Estatístico de Mann Whitney

A Tabela 4 traz os dados referentes à avaliação perceptivo-auditiva dos participantes do estudo.

Os PCQ exibiram uma intensidade do desvio vocal moderado, no grau geral para a emissão da vogal $/ \varepsilon /$ e fala. Os PSQ também possuíram um desvio vocal, mas de intensidade leve.

O grupo $P C Q$ possuiu em suas vozes a presença de rugosidade, soprosidade, tensão e instabilidade acima do esperado para vozes sem alteração, já os PSQ, apenas os parâmetros rugosidade e instabilidade se encontraram acima do esperado para vozes adaptadas.

De um modo geral, as vozes do grupo PCQ apresentaram-se mais alteradas, em todos os parâmetros, comparadas as do PSQ.

\section{DISCUSSÃO}

As disciplinas que compõem a área de Humanas foram predominantemente ministradas pelos professores que apresentaram queixas vocais. Acredita-se que essas disciplinas, geralmente, são as que apresentam conteúdo mais teórico, implicando, quase sempre, em aulas expositivas, aumentando a demanda vocal por parte dos professores, fato esse que pode maximizar o risco do aparecimento de algum problema vocal.

As escolas, em geral, não oferecem condições favoráveis ao desenvolvimento do trabalho dos docentes. Essas escolas não possuíam preocupação com a saúde vocal dos professores, inexistindo algum programa de prevenção dos distúrbios da voz ou ainda um programa de conservação/ promoção vocal. Inclusive quase todos os professores relataram que as salas de aula possuíam acústica desfavorável devido a presença de ruído interno e externo.
O ruído mostra-se como um competidor para a voz do professor na sala de aula, que o obriga a aumentar a intensidade da voz para ser ouvido pelos discentes, podendo gerar uma sobrecarga vocal que, posteriormente, pode levar ao desenvolvimento de alterações teciduais e/ou musculares na laringe. O ruído ambiental pode ser ainda um fator desencadeante do estresse de professores no trabalho, ocorrendo várias alterações fisiológicas, como cardiovasculares, intestinais e emocionais ${ }^{16}$.

Em uma recente pesquisa ${ }^{17}, 75 \%$ dos professores consideraram ruidoso o local onde se situam as salas em que ministram disciplinas. Relataram que o ruído ambiental fez com que $79,2 \%$ precisem falar alto, o que acontece frequentemente para $73,7 \%$ dos professores, sendo que $37,5 \%$ apresentaram rouquidão após as aulas e $29,2 \%$ pigarro.

O número de sintomas vocais foi usado como o critério de inclusão no grupo de professores com queixas vocais (PCQ) e professores sem queixas vocais (PSQ). Os PCQ expressaram sentir, no momento atual, um número igual ou superior a três sintomas, esse número pode ser indicativo de alguma alteração já instalada, por isso considerou-se um número preocupante, analisando que esse profissional depende, essencialmente, da sua voz para exercer sua função. Enquanto os PSQ apresentaram menos de um sintoma vocal.

Em pesquisa com professores e não professores, realizada nos Estados Unidos ${ }^{10}$, encontrou-se um número de 4,3 sinais e sintomas vocais e os não professores uma média de 3,1 , já no Brasil, estudo semelhante ${ }^{11}$ revelou um número de 3,5 sinais e sintomas vocais e os não professores 1,7.

Em recente estudo ${ }^{18}$ comparou-se o impacto de uma perda vocal entre não professores e professores, sendo estes últimos os que mais se 
preocupam com uma eventual perda dessa função, atribuindo mesmo valor de impacto para a incapacidade de se locomover (não andar), expondo a preocupação desses profissionais em relação à voz. Além de limitar a vida profissional dos professores, a voz também possui implicações nas relações sociais e emocionais, pois a partir dela pode se comunicar, expressar ideias e interagir com o meio a qual está inserido.

O domínio do QVV relacionado ao funcionamento físico foi de forma significante mais comprometido nos PCQ, concordando com o número de sintomas vocais. Esse resultado se mostra semelhante em alguns estudos ${ }^{3}$, demonstrando que sintomas físicos, como "cansaço ao falar", causam um maior impacto na qualidade de vida dos sujeitos, sendo, portanto, mais perceptíveis. Os PCQ demonstraram uma dificuldade para exercer sua função por causa da voz. Os escores para os PSQ mostraram uma boa relação entre a voz e a qualidade de vida desse grupo.

A sensação que o ar acaba rápido durante a emissão, pode evidenciar o mau fechamento glótico. A presença de uma fenda glótica aumenta o fluxo de ar transglótico, comprometendo a vibração das pregas vocais, fato esse que reflete em emissões curtas, com maior esforço e com episódios frequentes de recarga de ar durante a fala.

Outra comparação a ser destacada aparece no IDV, onde os escores dos PCQindicam que a desvantagem vocal possui interferência nas situações do cotidiano, o que acontece menos para os PSQ. O IDV orgânico pode sugerir alguma alteração nas estruturas responsáveis pela produção vocal, podendo estar comprometidas nos PCQ. Alguns sintomas, como "esforço para falar" e "fazer força para que a voz saia", reforçam uma dificuldade na coaptação glótica, sugerindo a presença de uma lesão de massa ou ajuste muscular inadequado, causando uma deficiência no fechamento das pregas vocais, e, assim, exigindo um esforço maior do sujeito para conseguir colocá-las em vibração ${ }^{9}$.

Apesar dos questionários de autoavaliação vocal sugerirem um comprometimento das vozes dos $P C Q$, esse grupo não possui a percepção dessa limitação, não admitindo que exista um problema na voz. Fazendo com que esse profissional não procure um especialista para ajustar a voz a sua demanda, continuando a realizar esforços vocais e agravando a sua alteração. Esse fato leva a crer que muitos professores acham que ter uma voz desviada/alterada é uma característica inerente a sua profissão.

O SRQ observa o grau de comprometimento emocional (estresse, ansiedade e depressão), estando mais comprometidos aqueles que afirmam sentir seis ou mais sintomas expostos no questionário. Os PCQ apresentaram um grau maior de comprometimento emocional, apontando um resultado próximo do esperado para pessoas com restrições emocionais. Já os $P S Q$ não apresentam um comprometimento emocional. Esse fato é confirmado nos escores do IDATE Estado, que mostram que os $\mathrm{PCQ}$ apresentam comprometimento emocional acima do esperado para pessoas ansiosas (acima de 41) 8,14. $^{\text {8. }}$

A ocorrência de sinais e sintomas vocais e emocionais em professores com e sem queixas está demonstrada na Tabela 3. A partir desta nota-se um grande número de relatos de sintomas vocais dos $P C Q$ em relação aos $P S Q$.

O sintoma "dor na garganta" e "garganta seca" se mostrou prevalente nos professores, tanto no passado como no presente. A falta de hidratação durante as aulas pode levar a sensação de garganta seca, destacando a falta de cuidados básicos com a voz, assim como o aumento do fluxo de ar transglótico também pode levar a essa sensação. Este sintoma também foi destaque em outro estudo juntamente com coceira na garganta e fadiga vocal ${ }^{19}$. Em outra pesquisa, a maioria afirma sentir a garganta seca/raspando (26 professores $61,90 \%)^{20}$.

Outros sintomas destacados na Tabela 3 são: problemas para falar baixo, problema para projetar a voz, problema para cantar agudo e voz monótona, o que indica uma dificuldade dos professores em flexibilizar a voz, mudar a frequência e alterar o tom.

A prevalência do sintoma gosto ácido na boca, interrogado no QSSV, pode indicar presença de refluxo laringofaríngeo. Este é reflexo de hábitos alimentares inadequados e alterações no estômago, fato esse que corrobora com o dado do SRQ, o sintoma má digestão. A alteração estomacal pode ser a gastrite, que, além de outros fatores, tem o emocional como causa. Nervosismo, estresse e ansiedade podem desenvolver a gastrite estomacal. O estresse provoca a estimulação da produção de ácido clorídrico, que corrói a parede do estômago podendo, também, afetar as pregas vocais $^{21}$. Confirmando mais um indício da presença de alterações emocionais nos professores pesquisados. Um estudo concluiu que o refluxo gastroesofageano pode estar relacionado com a mudança do epitélio da parede posterior da laringe, assim como também estar relacionado com alterações histológicas inflamatórias dessa região, ${ }^{22}$ estrutura essa responsável pela produção da voz.

De forma geral, analisou-se que os maiores índices de comprometimento emocional foram observados no grupo dos professores com queixa vocal. Os profissionais que relataram mais sintomas 
vocais no QSSV (Tabela 3), assim como uma menor qualidade de vida em voz e uma maior desvantagem vocal (Tabela 2), são os que obtiveram maiores escores nos questionários relacionados à emoção, tanto o SRQ como o IDATE-Estado.

$\mathrm{Na}$ emissão da vogal, as vozes apareceram mais alteradas comparando-as com a fala encadeada (contagem) na escala de avaliação. A avaliação da vogal fornece, predominantemente, dados da fonte glótica, enquanto que a fala possui maior participação dos ajustes supraglóticos, de filtro.

A partir da análise perceptivo-auditiva pôde-se perceber uma intensidade do desvio vocal moderado para os PCQ, na avaliação da vogal / $/$ /, podendo interferir na eficiência do seu trabalho e, se não cuidada, essa alteração pode se elevar, comprometendo a sua permanência na função que desempenha. Com isso, ressalta-se a importância desses profissionais manterem hábitos vocais saudáveis, tomando alguns cuidados e procurando especialistas, para realização de condicionamento vocal mínimo para o uso profissional da voz.

De acordo com os dados da Tabela 4, percebe-se que os PCQ apresentaram vozes com presença de rugosidade, soprosidade e tensão. Sabe-se que a rugosidade é a manifestação vocal mais comum, ocorrendo por irregularidade de vibração das pregas vocais ${ }^{9}$. Além disso, as vozes dos professores também apresentaram soprosidade, com a intensidade do desvio vocal moderado.

$\mathrm{Na}$ tensão, observa-se uma atividade dos músculos supra-hióideos, que podem, simultaneamente, elevar a laringe e fazer com que esse som seja produzido em uma frequência mais aguda, com uma projeção vocal reduzida e esforço na região laringofaríngea. $O$ uso repetitivo desse padrão poderá acarretar o desenvolvimento de uma lesão de massa, por comportamento e padrão vocal inadequado. Assim, a tensão gerada pela ansiedade pode acarretar modificações no trato vocal, sobrecarregando a ressonância e podendo desencadear um processo de alteração vocal ${ }^{8}$.
Por meio da análise da perceptivo-auditiva, pôde-se inferir que o grupo PCQ apresentou um desvio vocal considerável, o qual reflete na sua qualidade de vida, como destacado nos questionários de autoavaliação vocal. Esses dados evidenciaram uma relação entre problemas vocais e emocionais, sobretudo ansiedade. As vozes mais alteradas emanaram de sujeitos considerados mais ansiosos. Com isso verificou-se a necessidade de uma maior investigação da relação entre esses fatores, já que não se conseguiu inferir se o fator ansiedade é o causador de uma desordem vocal ou se a relação inversa também é válida.

É importante mencionar que a Paraíba não possui uma lei que regule e/ou possibilite a criação de um Programa de Saúde Vocal que possa proteger a comunicação dos docentes do Estado, como é o caso de alguns Estados e Municípios do Brasil que já possuem ${ }^{23}$. Sabe-se que iniciativas desse tipo podem, além de melhorar a saúde dos seus trabaIhadores, favorecer a relação entre professor-aluno, bem como facilitam o processo de aprendizado, tendo em vista que a voz é o instrumento principal para a transmissão de conhecimento do professor.

\section{CONCLUSÃO}

Os professores considerados com queixas vocais possuem um maior comprometimento emocional e vocal comparados aos professores que não apresentam queixas vocais, fato que indica a existência da interferência das emoções no desenvolvimento e/ou manutenção de desordens na voz. Esse fato é refletido no número de queixas relatadas e o comprometimento da qualidade de vida desses profissionais. Assim, destaca-se a importância de um programa de saúde vocal para os professores, para que estes tenham conhecimento sobre a voz e passem a manter cuidados de saúde vocal, evitando problemas futuros que podem culminar no comprometimento da qualidade de vida e afastamento do trabalho. 


\section{ABSTRACT}

Purpose: to evaluate the effect of emotional risk factors on the voice of teachers with and without vocal complaints. Method: the sample comprised 44 teachers. We used a form for collecting personal and professional data, three questionnaires concerning vocal aspects, and two questionnaires for data related to emotion. Further, we recorded voices for auditory-perceptual analysis. The teachers were divided based on the number of symptoms reported: 22 volunteers in a group of Teachers Without Complaints (TWOC) and 22 in a group of Teachers With Complaints (TWC). The latter group included individuals reporting more than three vocal symptoms. Results: the average number of symptoms described in the Voice Signs and Symptoms Questionnaire was $5.7( \pm 2.8)$ and $0.8( \pm 0.9)$ for the $P C Q$ and $P S Q$ groups, respectively. The PSQ reported better vocal self-assessment $(p=0.01)$ and the $P C Q$ reported greater vocal impairment $(p=0.001)$. The $P S Q$ group obtained the highest scores in the Physical Voice-related Quality of Life $(P-V Q L)(p=0.0007)$ and Total VQL $(p=0.0006)$. The PCQ had higher values in the Total Voice Handicap Index (VHI) $(p=0.0003)$ and Organic VHI $(p=$ 0.0006), and greater emotional impairment in the Self-Report Questionnaire [5.7 $( \pm 3.9)]$ and Trait-State Anxiety Inventory $[42.5( \pm 12.7)]$. Auditory-perceptual analysis showed that the PCQ had moderate vocal deviation with vocal roughness, breathiness, and tension, while the PSQ group showed slight deviation in all parameters. Conclusion: teachers with vocal complaints are exposed to more risk factors, and report more symptoms and vocal and emotional impairments.

KEYWORDS: Faculty; Voice; Anxiety; Emotions; Communication; Speech, Language and Hearing Sciences

\section{REFERÊNCIAS}

1. Servilha EAM, Pereira PM. Condições de trabalho, saúde e voz em professores universitários. Rev. Ciên. Med. 2008;17(1):21-31.

2. Grillo MHMM, Penteado RZ. Impacto da voz na qualidade de vida de professore(a)s do ensino fundamental. Pró-Fono R. Atual. Cient. 2005; 17(3):321-30.

3. Jardim R, Barreto SM, Assunção AA. Condições de trabalho, qualidade de vida e disfonia entre docentes. Cad. Saúde Pública. 2007;23(10):2439-61.

4. Azevedo LL, Vianello L, Oliveira HGP, Oliveira IA, Oliveira BFV, Silva CM. Queixas vocais e grau de disfonia em professoras do ensino fundamental. Rev Soc Bras Fonoaudiol. 2009;14(2):192-6.

5. Jung, CR. O estresse e a voz, [Monografia]. Porto Alegre: Centro de Especialização em Fonoaudiologia Clínica - CEFAC; 1999.

6. Ramig L, Verdolini K. Treatment efficacy: voice disorders. J. Speech Lang. Hear. Res. 1998;41(1):101-6.

7. Orlova OS, Vasilenko IS, Zakharova AF, Samokhvalova LO, Kozlova PA. The prevalence, causes and specific features of voice disturbances in teachers. Vestn. Otorrinolaringol. 2000;5:18-21.

8. Almeida AAF, Behlau M, Leite JR. Correlação entre ansiedade e performance comunicativa. Rev Soc Bras de Fonoaudiol. 2011;16(4):384-9.
9. Behlau M. Voz - O livro do especialista. Volume I. Rio de Janeiro: Editora Revinter, 2008.

10. Roy N, Merrill RM, Thibeault S, Gray SD, Smith EM. Voice disorders in teachers and the general population: effects on work performance, attendance, and future career choices. J Speech Lang Hear Res. 2004;47(3):542-51.

11. Zambon F, Behlau M, Roy N. Considerações Preliminares sobre um Levantamento Epidemiológico Brasileiro de Distúrbios Vocais em Professores. In: 36th Annual Symposium: Care of the Professional Voice. Philadelphia : The Voice Foundation, 2007.

12. Gasparini G, Behlau M. Quality of life: validation of the brazilian version of the voice-related quality of life (V-RQOL). J Voice. 2009;23:76-81.

13. Behlau M, Santos LMA, Oliveira G. Crosscultural adaptation and validation of the voice handicap index into brazilian Portuguese. J Voice. 2009;25(3):354-9.

14. Biaggio AMB, Natalício L. Manual para $\mathrm{O}$ Inventário de Ansiedade Traço-Estado (IDATE). Rio de Janeiro: Centro Editor de Psicologia Aplicada (CEPA); 1979.

15. Mari JJ, Williams P. A validity study of a Psychiatric Screening Questionnaire (SRQ-20) in primary care in the city of São Paulo. Br J Psychiatr 1986;148:23-6. 
16. Oiticica MLGR; Gomes, MLB O estresse do professor acentuado pela precariedade das condições acústicas das salas de aula. XXIV Encontro Nac. de Eng. de Produção - Florianópolis, SC, Brasil, 03 a 05 de nov de 2004.

17. Costa, DB. Lima, ILB; Almeida, LNA; Bandeira, RN; Andrade, WTLA. O ruído como agente dificultador da atuação dos professores em salas de aula de uma universidade pública do estado da Paraíba. 26ํㅡㄹ Encontro Internacional de Audiologia, Maceió-Al, Brasil, 17 a 20 de abril de 2010.

18. Park K, Behlau M. Perda da voz em professores e não professores. Rev Soc Bras Fonoaudiol. 2009;14(4):463-9.

19. Luchesi KF, Mourão LF, Kitamura S. Ações de promoção e prevenção à saúde vocal de professores: uma questão de saúde coletiva. Rev. Cefac. 2010;12(6): 945-53.
20. Silverio KCA, Gonçalves CGO, Penteado RZ, Vieira TPG, Libardi A, Rossi D. Ações em saúde vocal: proposta de melhoria do perfil vocal de professores. Pró-Fono R. Atual. Cient. 2008;20(3):177-82.

21. Silva EF. A voz dentro da relação psíquicoorgânica: estudo sobre a influência das emoções na voz do ator. R.cient./FAP. 2009;4(1):1-19.

22. Gomes GF, Filho EDM, Coelho JCU, Salvalaggio PRO, Sebastião APM, Suzana DG. Achados histológicos na parede posterior da laringe em pacientes com refluxo gastroesofageano (GERD). Rev. Bras. Otorrinolaringol. 2001;67(6):770-4.

23. Oliveira, IB Almeida AAF, Raize T. Voz Profissional: produção científica da Fonoaudiologia brasileira. São Paulo: Sociedade Brasileira de Fonoaudiologia, 2008. Disponível em <http://www. sbfa.org.br/portal/voz_profissional/index.htm> Acessado em Janeiro de 2012.

Recebido em: 12/07/2012

Aceito em: 01/04/2013

Endereço para correspondência:

Anna Alice Figueirêdo de Almeida

Universidade Federal da Paraíba, Centro de

Ciências da Saúde

Departamento de Fonoaudiologia

Cidade Universitária - Campus I - Castelo Branco

João Pessoa - PB - Brasil

CEP: $58051-900$

E-mail: anna_alice@uol.com.br 